\title{
Cumulant generating function formula of heat transfer in ballistic system with lead-lead coupling
}

\author{
Huanan Li - Bijay Kumar Agarwalla, and Jian-Sheng Wang \\ Department of Physics and Center for Computational Science and Engineering, \\ National University of Singapore, Singapore 117542, Republic of Singapore
}

(Dated: 24 Aug 2012)

\begin{abstract}
Based on two-time observation protocol, we consider heat transfer in a given time interval $t_{M}$ in lead-junction-lead system taking coupling between the leads into account. In view of the two-time observation, consistency conditions are carefully verified in our specific family of quantum histories. Furthermore, its implication is briefly explored. Then using nonequilibrium Green's function (NEGF) method, we obtain an exact formula for the cumulant generating function for heat transfer between the two leads valid in both transient and steady-state regimes. Also, a compact formula for the cumulant generating function in the long-time limit is derived, for which the Gallavotti-Cohen (GC) fluctuation symmetry is explicitly verified. In addition, a brief discussion of Di Ventra's trick regarding whether the effect of the repartitioning procedure of the total Hamiltonian on nonequilibium steady-state current fluctuation exists is given. All kinds of properties of nonequilibrium current fluctuations such as the fluctuation theorem in different time regimes could be readily given according to these exact formulas.
\end{abstract}

PACS numbers: 05.70.Ln, 05.40.-a, 44.10.+i, 65.80.-g

\section{INTRODUCTION}

The physics of nonequilibrium many-body systems is one of the most rapidly expanding areas of theoretical physics. In the combined field of non-equilibrium state and statistics, the distribution of transferred charges in electronic case or heat in phononic case, the so-called full counting statistics (FCS), plays an important role, according to which we could understand the general features of currents and their fluctuations. Also, it is well known that the noise generated by nanodevices contains valuable information on microscopic transport processes not available from only transient or steady current. In FCS, the key object we need to study is the cumulant generating function (CGF) which presents high order correlation information of the corresponding system for the transferred quantity.

The study of the FCS started from the field of electronic transport pioneered by Levitov and Lesovik, who presented an analytical result for the CGF in the longtime limit $\underline{1}$. After that, many works followed in electronic FCS $^{2-5}$, while much less attention is given to phonon transport. Saito and Dhar are the first ones to borrow this concept to thermal transport ${ }^{6}$. Later, Ren and his co-workers gave results for two-level systems ${ }^{7}$. And very recently, transient behavior and long-time limit of CGF have been obtained in lead-junction-lead harmonic networks both classically and quantum-mechanically using Langevin equation method and NEGF method, respectively $\stackrel{-10}{-10}$. Experimentally the FCS in electronic case has been carefully studied and the cumulants to very high order have been successfully measured in quantumdot systems ${ }^{11,12}$. In principle, similar measurements could be carried out for thermal transport, e.g., in a nanoresonator system.

Through modern nanoscale technology, small junction is easily realized such as in certain nanoscale systems, for instance, a single molecule or, in general, a small cluster of atoms between two bulk electrodes. In that case, the electrode surfaces of the bulk conductors may be separated by just a few angstroms so that some finite electronic coupling between the two surfaces is present taking into account the long-range interaction. In order to study steady current in this situation, Di Ventra suggest that we can choose our "sample" region (junction) to extend several atomic layers inside the bulk electrodes where screening is essentially complete so that the above coupling could be negligible 13 . This trick has been checked in some restricted case in a recent work ${ }^{14}$. However, whether this intuitively reasonable trick applies to all the higher cumulants of heat transfer in steady state is still a question. Therefore we will briefly discuss this question at the end of the article. And obviously this trick can not be applied to study transient behavior of all the cumulants of heat transfer. Thus, in this work, we want to construct the CGF of heat transfer in a general case taking into account the coupling between the lead explicitly, which include the information of all the cumulant of heat transfer. Also, the transient behavior and the long-time limit of CGF of heat transfer would be dealt with on an equal footing based on the NEGF method.

This paper is organized as follows. We start in Sec. II by introducing the model and the consistent quantum framework. And then we employ this framework to derive the CGF of heat transfer across the junction in Sec. III] In addition, we obtain a compact form of the long-time limit of the CGF in Sec. IV] based on which the steady-state fluctuation theorem (SSFT) is checked and the generalized Caroli formula closely related to the first cumulant is recovered in Sec. V At the end, we will briefly discuss Di Ventra's trick. 


\section{MODEL AND CONSISTENT QUANTUM FRAMEWORK}

We consider the lead-junction-lead model initially prepared in product state $\rho^{i n i}=\frac{e^{-\beta_{L} H_{L}}}{\operatorname{Tr}\left(e^{-\beta_{L} H_{L}}\right)} \otimes \frac{e^{-\beta_{C} H_{C}}}{\operatorname{Tr}\left(e^{-\beta_{C} H_{C}}\right)} \otimes$ $\frac{e^{-\beta_{R} H_{R}}}{\operatorname{Tr}\left(e^{-\beta_{R} H_{R}}\right)}$. We can imagine that left lead $(L)$, center junction $(C)$, and right lead $(R)$ in this model were in contact with three different heat baths at the inverse temperatures $\beta_{L} \equiv\left(k_{B} T_{L}\right)^{-1}, \beta_{C} \equiv\left(k_{B} T_{C}\right)^{-1}$ and $\beta_{R} \equiv\left(k_{B} T_{R}\right)^{-1}$ respectively, for time $t<0$. At time $t=0$, all the heat bath are removed, and coupling of the center junction with the leads $H_{L C} \equiv u_{L}^{T} V^{L C} u_{C}$ and $H_{C R} \equiv u_{C}^{T} V^{C R} u_{R}$ and the lead-lead coupling term $H_{L R}=u_{L}^{T} V^{L R} u_{R}$ are switched on abruptly. Now the total Hamiltonian of the lead-junction-lead system will become

$$
H_{\text {tot }}=H_{L}+H_{C}+H_{R}+H_{L C}+H_{C R}+H_{L R},
$$

where $H_{\alpha}=\frac{1}{2} p_{\alpha}^{T} p_{\alpha}+\frac{1}{2} u_{\alpha}^{T} K^{\alpha} u_{\alpha}, \alpha=L, C, R$, represents coupled harmonic oscillators, $u_{\alpha} \equiv \sqrt{m} x_{\alpha}$ and $p_{\alpha}$ are column vectors of transformed coordinates and corresponding conjugate momenta in region $\alpha$. The superscript $T$ stands for matrix transpose.

In order to extract information on heat transfer, we introduce two-time observation protocol in the process of the evolution of the system; that is, at time $t=0^{-}$, we carry out the measurement of energy of the left lead associated with the operator $H_{L}$, obtaining the result to be the eigenvalue $a$ of $H_{L}$, and measure it again at time $t=t_{M}$, obtaining the eigenvalue $b$ of $H_{L}$. Here the measurement is in the sense of quantum measurement of von Neumann $\underline{15}$. This quantum history $\underline{\underline{16}}$, that is a sequence of quantum events at successive times, is represented by the product projector $Y=P_{0^{-}}^{a} \odot P_{t_{M}}^{b}$, where $\odot$ is a variant of the tensor product symbol $\otimes$, emphasizing that the factors in the quantum history refer to different times, and $P_{0^{-}}^{a}$ is the projector on the energy eigenstate of $H_{L}$ with energy a measured at time $t=0^{-}$, similarly for $P_{t_{M}}^{b}$. And the corresponding chain operator $K(Y)$ is given by the expression $K(Y)=P_{t_{M}}^{b} U\left(t_{M}, 0^{-}\right) P_{0^{-}}^{a}$ in the case of a quantum history $Y=P_{0^{-}}^{a} \odot P_{t_{M}}^{b}$ involving just two times, where $U\left(t_{M}, 0^{-}\right)$is the time evolution operator of the full Hamiltonian $H_{\text {tot }}$. It could be easily verified that the joint probability distribution for the quantum history $Y$ is

$$
\operatorname{Pr}(Y)=\left\langle K^{\dagger} K\right\rangle \equiv \operatorname{Tr}\left\{\rho^{i n i} K^{\dagger} K\right\},
$$

where the superscript $\dagger$ stands for transpose conjugate and from now on the angle brackets $\langle\ldots\rangle$ simply denotes ensemble average with respect to $\rho^{i n i}$. Here it is worth mentioning that this scheme for the joint probability distribution could be readily extended to many-time measurement.

Taking the commutator $\left[\rho^{i n i}, P_{0^{-}}^{a}\right]=0$ into account, we can verify that consistency conditions $\left\langle K(Y), K\left(Y^{\prime}\right)\right\rangle_{\rho^{i n i}} \equiv \operatorname{Tr}\left\{\rho^{i n i} K(Y)^{\dagger} K\left(Y^{\prime}\right)\right\}=0$ for all $Y \neq Y^{\prime}$ are fulfilled, which guarantees that we are working with a consistent quantum framework. The consistent quantum framework, combining the axiom of probability with the Born rule of the quantum theory, is crucial to understand the probability aspect of the quantum theory $\underline{16}$. Now we want to gain some insight into the specific consistent quantum framework we study from thermal transport point of view.

As we will consider later, the generating function (GF) for heat transfer during the time $t_{M}$ is defined as

$$
\mathcal{Z}(\xi)=\sum_{a, b} e^{i \xi(a-b)} \operatorname{Pr}\left(P_{0^{-}}^{a} \odot P_{t_{M}}^{b}\right),
$$

where the summation for $a$ and $b$ extends over all the eigenvalues of $H_{L}$. Then based on this definition, we simply take the derivative of $\mathcal{Z}(\xi)$ with respect to $i \xi$ and then set $\xi=0$ to obtain the average heat transfer out of left lead $L$

$$
\begin{aligned}
& Q_{L}\left(t_{M}\right) \\
= & \sum_{a, b}(a-b) \operatorname{Pr}\left(P_{0^{-}}^{a} \odot P_{t_{M}}^{b}\right) \\
= & \operatorname{Tr}\left[\rho^{i n i} H_{L}\right]-\operatorname{Tr}\left[\rho^{i n i} U\left(0^{-}, t_{M}\right) H_{L} U\left(t_{M}, 0^{-}\right)\right],
\end{aligned}
$$

where properties $\left(P_{0^{-}}^{a}\right)^{2}=P_{0^{-}}^{a}$ and $\sum_{a} P_{0^{-}}^{a}=1$ for the projector $P_{0^{-}}^{a}$, and similarly for $P_{t_{M}}^{b}$ are used. Also we use the key relation $\left[\rho^{i n i}, P_{0^{-}}^{a}\right]=0$, which assures that we are working with a consistent quantum framework. Thus, we immediately realize that

$$
\frac{d Q_{L}\left(t_{M}\right)}{d t_{M}}=-\left\langle\frac{d H_{L}\left(t_{M}\right)}{d t_{M}}\right\rangle,
$$

of which the right-hand side is just the natural definition of thermal current $I_{L}$ out of the left lead at time $t=t_{M}$. Actually, if the initial density matrix does not commute with $P_{0^{-}}^{a}$, such as a steady-state density matrix, so that the framework we work with is not consistent, then the thermal current deriving from GF for the heat transfer will not be equal to the natural definition of it.

\section{CUMULANT GENERATING FUNCTION}

As was mentioned before, we proceed to study the GF for the heat transfer out of the left lead. According to the definition of the GF in Eq. (3), we can obtain

$$
\mathcal{Z}(\xi)=\left\langle U_{\xi / 2}\left(0^{-}, t_{M}\right) U_{-\xi / 2}\left(t_{M}, 0^{-}\right)\right\rangle,
$$

where $U_{-\xi / 2}\left(t_{M}, 0^{-}\right)$means evolution operator associated with the modified total Hamiltonian $H_{t o t}^{-\xi / 2} \equiv e^{i(-\xi / 2) H_{L}} H_{t o t} e^{-i(-\xi / 2) H_{L}}$, and similarly for $U_{\xi / 2}\left(0^{-}, t_{M}\right)$. Transforming to the interaction picture with respect to the free part of the modified 
total Hamiltonian $H_{0}=H_{L}+H_{C}+H_{R}$, the GF for the heat transfer becomes

$$
\begin{aligned}
& \mathcal{Z}(\xi)= \\
& \left\langle T_{\tau} e^{-\frac{i}{\hbar} \int_{C} d \tau \hat{u}_{L}\left(\hbar x_{\tau}+\tau\right)\left(V^{L C} \hat{u}_{C}(\tau)+V^{L R} \hat{u}_{R}(\tau)\right)+\hat{u}_{C}(\tau) V^{C R} \hat{u}_{R}(\tau)}\right\rangle
\end{aligned}
$$

where $T_{\tau}$ is a $\tau$-ordering operator arranging operators with earliest $\tau$ on the contour $C$ (from $0^{-}$to $t_{M}$ and back to $0^{-}$) to the right, and a caret is put above operators to denote their $\tau$ dependence with respect to the free Hamiltonian such as $\hat{u}_{C}(\tau)=e^{\frac{i}{\hbar} H_{C} \tau} u_{C} e^{-\frac{i}{\hbar} H_{C} \tau}$, and $x_{\tau}=-\xi / 2$ with $\tau=t^{+}$on the upper branch of the contour $C$, while $x_{\tau}=+\xi / 2$ with $\tau=t^{-}$on the lower branch.

The key step to evaluate GF is to rewrite the exponent in Eg. (8) as $-\frac{i}{\hbar} \int_{C} d \tau_{1} d \tau_{2} \frac{1}{2} u^{T}\left(\tau_{1}\right) V\left(\tau_{1}, \tau_{2}\right) u\left(\tau_{2}\right)$, where

$$
\begin{gathered}
u^{T}(\tau)=\left[\begin{array}{lll}
\hat{u}_{L}\left(\hbar x_{\tau}+\tau\right) & \hat{u}_{C}(\tau) & \hat{u}_{R}(\tau)
\end{array}\right], \\
V\left(\tau_{1}, \tau_{2}\right)=\left[\begin{array}{ccc}
0 & V^{L C} & V^{L R} \\
V^{C L} & 0 & V^{C R} \\
V^{R L} & V^{R C} & 0
\end{array}\right] \delta\left(\tau_{1}, \tau_{2}\right), \\
\left(V^{T}\left(\tau_{1}, \tau_{2}\right)=V\left(\tau_{1}, \tau_{2}\right)\right) .
\end{gathered}
$$

Here the generalized $\delta$-function $\delta\left(\tau_{1}, \tau_{2}\right)$ is simply counterpart of the ordinary Dirac delta function on the contour $C$, see, for example, Ref ${ }^{17}$.

Then expanding the exponential to perform a perturbation expansion and employing Feynman diagrammatic technique, especially Wick's theorem and the linked cluster theorem, the CGF for the heat transfer can be obtained to be

$$
\begin{aligned}
\ln \mathcal{Z}(\xi) & =\frac{1}{2} \sum_{n=1}^{\infty} \operatorname{Tr}_{(j, \tau)}\left[\frac{1}{n}\left(V g^{x}\right)^{n}\right] \\
& =\frac{1}{2} \sum_{n=1}^{\infty} \operatorname{Tr}_{(j, \tau)}\left[\frac{1}{n}\left(V_{r e d} g_{r e d}^{x}\right)^{n}\right] \\
& =-\frac{1}{2} \operatorname{Tr} \ln \left(I-\tilde{V}_{\text {red }} \tilde{g}_{r e d}^{x}\right)
\end{aligned}
$$

where in the first equality

$$
\begin{aligned}
& g^{x}\left(\tau_{1}, \tau_{2}\right) \\
= & {\left[\begin{array}{ccc}
g^{L}\left(\hbar x_{\tau_{1}}+\tau_{1}, \hbar x_{\tau_{2}}+\tau_{2}\right) & 0 & 0 \\
0 & g^{C}\left(\tau_{1}, \tau_{2}\right) & 0 \\
0 & 0 & g^{R}\left(\tau_{1}, \tau_{2}\right)
\end{array}\right], }
\end{aligned}
$$

with the equilibrium contour-ordered Green functions

$$
\begin{gathered}
g_{j k}^{\alpha}\left(\tau_{1}, \tau_{2}\right)=-\frac{i}{\hbar} \operatorname{Tr}\left\{\frac{e^{-\beta_{\alpha} H_{\alpha}}}{\operatorname{Tr}\left(e^{-\beta_{\alpha} H_{\alpha}}\right)} T_{\tau}\left[\hat{u}_{j}^{\alpha}\left(\tau_{1}\right) \hat{u}_{k}^{\alpha}\left(\tau_{2}\right)\right]\right\}, \\
\alpha=L, C, R
\end{gathered}
$$

and the notation $\operatorname{Tr}_{(j, \tau)}$ means trace both in real space index $j$ and contour time $\tau$ such as $\operatorname{Tr}_{(j, \tau)}\left[V g^{x}\right]=$ $\int_{C} d \tau_{1} \int_{C} d \tau_{2} \operatorname{Tr}_{j}\left[V\left(\tau_{1}, \tau_{2}\right) g^{x}\left(\tau_{2}, \tau_{1}\right)\right] ; \quad$ in the second equality, considering the structure of the last expression, instead of the full matrix we only need the finite reduced $\gamma$ 'ones, which make all the contribution to $\ln \mathcal{Z}(\xi)$, and

$$
V_{\text {red }}\left(\tau_{1}, \tau_{2}\right)=\delta\left(\tau_{1}, \tau_{2}\right)\left[\begin{array}{ccc}
0 & V_{\text {red }}^{L C} & V_{\text {red }}^{L R} \\
V_{\text {red }}^{C L} & 0 & V_{\text {red }}^{C R} \\
V_{\text {red }}^{R L} & V_{\text {red }}^{R C} & 0
\end{array}\right]
$$

is the generalized $\delta$-function $\delta\left(\tau_{1}, \tau_{2}\right)$ times the reduced total coupling matrix obtained by deleting all the zero column and row vectors of the full one except for the possible zero vectors whose row or column indexes are the center (junction) ones, and $g_{\text {red }}^{x}$ is the corresponding submatrix of $g^{x}$ just like $V_{\text {red }}$ of $V$; in the third equality a tilde above matrix means discretized contour-time version of the corresponding quantity such as $\left[\tilde{g}_{r e d}^{x}\right]_{\tau_{i} l, \tau_{j} n}=$ $\left[g_{r e d}^{x}\right]_{l n}\left(\tau_{i}, \tau_{j}\right) d \tau_{j}$ with an evenly spaced grid $\tau_{i}$ and $\tau_{j}$ along the contour $C$ and $I$ is the identity matrix.

Introducing the Dyson equation

$$
\begin{aligned}
& G\left(\tau, \tau^{\prime}\right) \\
= & g\left(\tau, \tau^{\prime}\right)+\int_{C} d \tau_{1} \int_{C} d \tau_{2} g\left(\tau, \tau_{1}\right) V\left(\tau_{1}, \tau_{2}\right) G\left(\tau_{2}, \tau^{\prime}\right) \\
= & g\left(\tau, \tau^{\prime}\right)+\int_{C} d \tau_{1} \int_{C} d \tau_{2} G\left(\tau, \tau_{1}\right) V\left(\tau_{1}, \tau_{2}\right) g\left(\tau_{2}, \tau^{\prime}\right),
\end{aligned}
$$

which actually defines $G=\left[\begin{array}{lll}G^{L L} & G^{L C} & G^{L R} \\ G^{C L} & G^{C C} & G^{C R} \\ G^{R L} & G^{R C} & G^{R R}\end{array}\right]$ based on $g\left(\tau, \tau^{\prime}\right)=g^{x \equiv 0}\left(\tau, \tau^{\prime}\right)$, we easily realized that

$$
\begin{aligned}
\tilde{G}_{r e d} & =\tilde{g}_{r e d}+\tilde{g}_{r e d} \tilde{V}_{r e d} \tilde{G}_{r e d} \\
& =\tilde{g}_{r e d}+\tilde{G}_{r e d} \tilde{V}_{r e d} \tilde{g}_{r e d}
\end{aligned}
$$

still holds. And from now on, for notational simplicity, we omit all the subscript red with the understanding that these matrices are of finite dimensions in the real space domain. Thus, employing Egs. (19) and (20) along with the equality $\operatorname{Tr} \ln A=\ln \operatorname{det} A$, we obtain

$$
\begin{gathered}
\ln \mathcal{Z}(\xi)=-\frac{1}{2} \ln \operatorname{det}(I-d), \\
d \equiv\left[\begin{array}{ll}
\tilde{V}^{L C} & \tilde{V}^{L R}
\end{array}\right]\left[\begin{array}{ll}
\tilde{G}^{C C} & \tilde{G}^{C R} \\
\tilde{G}^{R C} & \tilde{G}^{R R}
\end{array}\right]\left[\begin{array}{c}
\tilde{V}^{C L} \\
\tilde{V}^{R L}
\end{array}\right] \tilde{g}_{L}^{A},
\end{gathered}
$$

where $\tilde{g}_{L}^{A}$ is the discretized contour-time version of $g_{L}^{A}\left(\tau_{1}, \tau_{2}\right)=g^{L}\left(\hbar x_{\tau_{1}}+\tau_{1}, \hbar x_{\tau_{2}}+\tau_{2}\right)-g^{L}\left(\tau_{1}, \tau_{2}\right)$.

If we introduce $\tilde{g}_{L}^{-1}$ satisfying $\tilde{g}_{L}^{-1} \tilde{g}_{L}=I$ and employ Eqs. (19) and (20), we can simplify the CGF for heat transfer further to be

$$
\ln \mathcal{Z}(\xi)=-\frac{1}{2} \ln \operatorname{det}\left(I-\tilde{g}_{L}^{-1}\left(\tilde{G}^{L L}-\tilde{g}_{L}\right) \tilde{g}_{L}^{-1} \tilde{g}_{L}^{A}\right),
$$

which is valid in both transient and steady-state regimes. 


\section{THE STEADY-STATE CGF}

Now, we proceed to evaluate the long-time limit of the CGF in Eg. (22) called the steady-state CGF. Transforming Eg. (22) from contour-time to real-time and then using the Keldysh rotation, which is essentially an orthogonal transformation that

$$
\begin{aligned}
\breve{A}\left(t_{1}, t_{2}\right) & =O^{T} \sigma_{z}\left[\begin{array}{ll}
A\left(t_{1}^{+}, t_{2}^{+}\right) & A\left(t_{1}^{+}, t_{2}^{-}\right) \\
A\left(t_{1}^{-}, t_{2}^{+}\right) & A\left(t_{1}^{-}, t_{2}^{-}\right)
\end{array}\right] O \\
& \equiv\left[\begin{array}{ll}
A^{r}\left(t_{1}, t_{2}\right) & A^{K}\left(t_{1}, t_{2}\right) \\
A^{\bar{K}}\left(t_{1}, t_{2}\right) & A^{a}\left(t_{1}, t_{2}\right)
\end{array}\right]
\end{aligned}
$$

with $O=\frac{1}{\sqrt{2}}\left[\begin{array}{cc}1 & 1 \\ -1 & 1\end{array}\right]$ and the Pauli $z$ matrix $\sigma_{z}=$ $\left[\begin{array}{cc}1 & 0 \\ 0 & -1\end{array}\right]$ appearing due to the transition from contourtime to real-time, see, Eg. Ref. ${ }^{10}$, we obtain

$$
\ln \mathcal{Z}(\xi)=\frac{1}{2} \sum_{n=1}^{\infty} \operatorname{Tr}_{(j, t)}\left[\frac{1}{n}\left(\breve{g}_{L}^{-1}\left(\breve{G}^{L L}-\breve{g}_{L}\right) \breve{g}_{L}^{-1} \breve{g}_{L}^{A}\right)^{n}\right]
$$

where the notation $\operatorname{Tr}_{(j, t)}$ means trace both in real space $j$ and real time $t$ such as $\operatorname{Tr}_{(j, t)}(\breve{A} \breve{B})=$ $\int_{0}^{t_{M}} d t_{1} \int_{0}^{t_{M}} d t_{2} \operatorname{Tr}_{j}\left[\breve{A}\left(t_{1}, t_{2}\right) \breve{B}\left(t_{2}, t_{1}\right)\right]$.

Before proceeding, a significant consideration comes that all kinds of real-time versions of the contour-time Green function $G\left(\tau, \tau^{\prime}\right)$ defined in Eqs. (17) or (18) are not necessarily time translationally invariant so that $\breve{G}^{L L}\left(t, t^{\prime}\right)$ may not simply depend on the time difference $t-t^{\prime}$. However, in the long-time limit, i.e., $t_{M} \rightarrow \infty$, the time translationally invariant part obtained from the lowest order of the Wigner transformation will dominate the $\mathrm{CGF}^{18}$. It is equivalent to saying that $\breve{G}^{L L}\left(t, t^{\prime}\right)=$ $\breve{G}^{L L}\left(t-t^{\prime}\right)$ is time translationally invariant in the longtime limit (higher order terms of the product of the Wigner transformation have been ignored).

Consequently, setting $t_{M} \rightarrow \infty$, and Fourier transforming Eq. (25), we get

$$
\begin{aligned}
& \ln \mathcal{Z}(\xi) \\
= & -\frac{1}{2} t_{M} \int_{-\infty}^{\infty} \frac{d \omega}{2 \pi} \ln \operatorname{det}\left(I-\left(\breve{g}_{L}^{-1} \breve{G}^{L L}-I\right) \breve{g}_{L}^{-1} \breve{g}_{L}^{A}\right),
\end{aligned}
$$

where,

$$
\begin{gathered}
\breve{g}_{L}=\left[\begin{array}{cc}
g_{L}^{r} & g_{L}^{K} \\
0 & g_{L}^{a}
\end{array}\right], \breve{G}^{L L}=\left[\begin{array}{cc}
G_{L L}^{r} & G_{L L}^{K} \\
0 & G_{L L}^{a}
\end{array}\right], \\
\breve{g}_{L}^{A}=\frac{1}{2}\left[\begin{array}{cc}
a-b & a+b \\
-a-b & -a+b
\end{array}\right], \\
a \equiv g_{L}^{>}\left(e^{-i \hbar \omega \xi}-1\right), b \equiv g_{L}^{<}\left(e^{i \hbar \omega \xi}-1\right)
\end{gathered}
$$

are all in frequency space. For NEGF notations and some general relations among Green's functions, we refer to Ref. 17.
To further simplify the steady-state CGF in Eq. (26), we use the formula $\operatorname{det}\left(\begin{array}{ll}A & B \\ C & D\end{array}\right)=\operatorname{det}(A D-B C)$ in case of $[C, D]=0$ to reduce the dimension of the matrix inside determinant by half. Therefore, the steady-state CGF is given by

$$
\begin{gathered}
\ln \mathcal{Z}(\xi)=-t_{M} \int_{-\infty}^{\infty} \frac{d \omega}{4 \pi} \ln \operatorname{det}\left\{I-\mathcal{T}_{\mathcal{G}}[\omega]\right. \\
\left.\times\left[\left(e^{i \xi \hbar \omega}-1\right) f_{L}\left(1+f_{R}\right)+\left(e^{-i \xi \hbar \omega}-1\right) f_{R}\left(1+f_{L}\right)\right]\right\},
\end{gathered}
$$

where $\mathcal{T}_{\mathcal{G}}[\omega] \equiv G_{L R}^{r} \tilde{\Gamma}_{R} G_{R L}^{a} \tilde{\Gamma}_{L}$ with

$$
\tilde{\Gamma}_{\{L, R\}} \equiv i\left[\left(g_{\{L, R\}}^{a}\right)^{-1}-\left(g_{\{L, R\}}^{r}\right)^{-1}\right]
$$

is the transmission matrix and $f_{\{L, R\}}=$ $\left\{\exp \left(\beta_{\{L, R\}} \hbar \omega\right)-1\right\}^{-1}$ is the Bose-Einstein distribution function for phonons.

In deriving it, we have used fluctuation dissipation theorem $e^{-\beta_{L} \hbar \omega} g_{L}^{>}[\omega]=g_{L}^{<}[\omega]=f_{L}\left(g_{L}^{r}-g_{L}^{a}\right)$ along with

$$
\begin{aligned}
& G_{L L}^{<} \\
= & G_{L L}^{r}\left(-i f_{L} \tilde{\Gamma}_{L}\right) G_{L L}^{a}+G_{L R}^{r}\left(-i f_{R} \tilde{\Gamma}_{R}\right) G_{R L}^{a}, \\
& G_{L L}^{r}-G_{L L}^{a}-G_{L L}^{r}\left(g_{L}^{a-1}-g_{L}^{r-1}\right) G_{L L}^{a} \\
= & G_{L R}^{r}\left(g_{R}^{a-1}-g_{R}^{r-1}\right) G_{R L}^{a},
\end{aligned}
$$

due to $\left(g_{C}^{a}\right)^{-1}-\left(g_{C}^{r}\right)^{-1}=0$ and the Langreth theorem ${ }^{18}$ acting on Eqs. (19) and (20). A computationally practical closed equation for $G_{L R}^{r}$ could be found in Ref ${ }^{14}$. The formulas Eq. (29) and Eq. (22) are our central results.

Now we recover the classical version of the CGF for the heat transfer in harmonic networks without the leadlead coupling, which was first derived in Ref. $\underline{8}$ using the Langevin equation method. To this end we simply set the lead-lead coupling $V^{L R}=0$ in Eqs. (19) and (20), then use the Langreth theorem and the Fourier transformation to obtain $G_{L R}^{r}=g_{L}^{r} V^{L C} G_{C R}^{r}=g_{L}^{r} V^{L C} G_{C C}^{r} V^{C R} g_{R}^{r}$ along with $G_{R L}^{a}=\left(G_{L R}^{r}\right)^{\dagger}$.

After setting $\hbar \rightarrow 0$ and employing $G_{R L}^{a}[-\omega]=$ $\left(G_{L R}^{r}[\omega]\right)^{T}$ and $\tilde{\Gamma}_{\{L, R\}}[-\omega]=-\tilde{\Gamma}_{\{L, R\}}[\omega]^{T}$, we can get

$$
\begin{aligned}
\lim _{t_{M} \rightarrow \infty} \frac{\ln \mathcal{Z}(\xi)}{t_{M}}= & -\int_{0}^{\infty} \frac{d \omega}{2 \pi} \ln \operatorname{det}\{I-\mathcal{T}[\omega] \\
& \left.\times k_{B}^{2} T_{L} T_{R}(i \xi)\left[i \xi+\left(\beta_{R}-\beta_{L}\right)\right]\right\},
\end{aligned}
$$

with

$$
\begin{aligned}
\mathcal{T}[\omega] & \equiv G_{C C}^{r} \Gamma_{R} G_{C C}^{a} \Gamma_{L}, \\
\Gamma_{\{L, R\}} & =i\left[\Sigma_{\{L, R\}}^{r}-\Sigma_{\{L, R\}}^{a}\right] \\
\Sigma_{\alpha}^{\{r, a\}} & =V^{C \alpha} g_{\alpha}^{\{r, a\}} V^{\alpha C}, \alpha=L, R .
\end{aligned}
$$




\section{THE SSFT AND CUMULANTS}

According to the steady-state CGF in Eq. (29), one could easily verify that the GC fluctuation symmetry 19 $\mathcal{Z}(\xi)=\mathcal{Z}\left(-\xi+i\left(\beta_{R}-\beta_{L}\right)\right)$ is still satisfied in this general set-up with lead-lead coupling. And recall the definition of GF in Eq. (3), we know that the probability distribution for the heat transferred $Q_{L}$ is $\operatorname{Pr}\left(Q_{L}\right)=$ $\frac{1}{2 \pi \delta(0)} \int_{-\infty}^{\infty} d \xi \mathcal{Z}(\xi) e^{-i \xi Q_{L}}$. Therefore, following the GC symmetry is the $\operatorname{SSFT} \operatorname{Pr}\left(Q_{L}\right)=e^{\left(\beta_{R}-\beta_{L}\right) Q_{L}} \operatorname{Pr}\left(-Q_{L}\right)$, which quantifies the violation of the second-law in the sense of probability.

Also, the CGF can be used to evaluate cumulants. Here we only focus on steady-state cumulants of heat transfer. As illustrated in Sec. III the steady current is closed related to the first cumulant so that

$$
\begin{aligned}
I_{L}^{s s} & =\lim _{t_{M} \rightarrow \infty} \frac{d}{d t_{M}}\left(\left.\frac{\partial \ln \mathcal{Z}(\xi)}{\partial(i \xi)}\right|_{\xi=0}\right) \\
& =\int_{0}^{\infty} \frac{d \omega}{2 \pi} \hbar \omega\left(f_{L}-f_{R}\right) \operatorname{Tr} \mathcal{T}_{\mathcal{G}}[\omega]
\end{aligned}
$$

where, $\mathcal{Z}(0)=1$ is used. This generalized Caroli formula with lead-lead coupling was given very recently in Ref 14 based on the definition of current directly, which gave us some valuable hints on the form of the steady-state CGF. The second cumulant describing the fluctuation of the heat transferred is obtained by taking the second derivative of steady-state CGF with respect to $i \xi$ and then setting $\xi=0$, which is

$$
\begin{aligned}
\left\langle\left\langle Q_{L}^{2}\right\rangle\right\rangle= & t_{M} \int_{-\infty}^{\infty} \frac{d \omega}{4 \pi}(\hbar \omega)^{2}\left\{\left(f_{L}+f_{R}+2 f_{L} f_{R}\right) \operatorname{Tr} \mathcal{T}_{\mathcal{G}}\right. \\
& \left.+\left(f_{L}-f_{R}\right)^{2} \operatorname{Tr} \mathcal{T}_{\mathcal{G}}^{2}\right\} .
\end{aligned}
$$

Higher-order cumulants are also systematically given by corresponding higher-order derivatives.

After some experiences on first few order cumulants of heat transfer, we want to discuss the trick suggested by Di Ventra mentioned in Sec. I that repartitioning the total Hamiltonian to avoid the inevitable coupling between leads in real nanoscale or mesoscopic system when calculating steady current. Now whether this trick is applicable to the evaluation of higher-order cumulant (fluctuation) of the heat transfer in steady state boils down to checking whether $\operatorname{Tr} \mathcal{T}_{G \text {,old }}^{n}=\operatorname{Tr} \mathcal{T}_{G, \text { new }}^{n}$ hold for all $n$ less than or equal to the corresponding order of the cumulant one wants, where $\mathcal{T}_{G, \text { old }}\left(\mathcal{T}_{G, \text { new }}\right)$ is the transmission matrix before (after) repartitioning the total Hamiltonian. Though giving a general verification is difficult, $\operatorname{Tr} \mathcal{T}_{G \text {,old }}^{n}=\operatorname{Tr} \mathcal{T}_{G, \text { new }}^{n}, \forall n$ is indeed true in a onedimensional central ring model, in which there is only one particle in the center junction connected with two semiinfinite spring chain leads and the interaction between the two nearest particles in the two leads respectively exists (in this case, both $\mathcal{T}_{G \text {,old }}$ and $\mathcal{T}_{G, \text { new }}$ are just a number). One step forward, if one think of CGF of heat transfer as the complete knowledge of the steady state, we can claim that the steady state is partition-independent after verification of $\operatorname{Tr} \mathcal{T}_{G \text {,old }}^{n}=\operatorname{Tr} \mathcal{T}_{G, \text { new }}^{n}, \forall n$ or equivalently $\ln \mathcal{Z}_{\text {old }}(\xi)=\ln \mathcal{Z}_{\text {new }}(\xi)$ in Eq. (29). Then we can partly answer a question raised by Caroli et al. regarding the (non)equivalence between the partitioned and partitionfree approaches 20 , which recently was partly settled by explicitly constructing a non-equilibrium steady state through adiabatically turning on an electrical bias between the leads 21 .

\section{SUMMARY}

We examine the statistics of heat transfer during time $t_{M}$ in a general lead-junction-lead quantum system, in which coupling between leads has been taken into account. To this end, a consistent quantum framework was introduced to derive the CGF valid in both transient and long-time regimes using the NEGF method. Also, the implication of consistency of the quantum framework was briefly discussed from thermal transport point of view. After that, a compact form of the steady-state CGF was obtained, following which the GC symmetry and the SSFT was verified. In addition, first few cumulants were given and generalized Caroli formula was recovered. Furthermore, some valuable hints with respect to the rigorous proof for whether fluctuation of heat transfer in steady state is partition-independent have been offered.

\section{ACKNOWLEDGMENTS}

We thank Juzar Thingna and Lifa Zhang for insightful discussions. This work is supported in part by URC Grant No. R-144-000-257-112.
* g0900726@nus.edu.sg

1 L. S. Levitov and G. B. Lesovik, JETP Lett. 58, 230 (1993)

2 W. Belzig and Y. V. Nazarov, Phys. Rev. Lett. 87, 197006 (2001)

3 Y. V. Nazarov and M. Kindermann, Eur. Phys. J. B 35, $413(2003)$
4 K. Schönhammer, Phys. Rev. B 75, 205329 (2007)

5 M. Esposito, U. Harbola, and S. Mukamel, Rev. Mod. Phys. 81, 1665 (2009)

6 K. Saito and A. Dhar, Phys. Rev. Lett. 99, 180601 (2007)

7 J. Ren, P. Hänggi, and B. Li, Phys. Rev. Lett. 104, 170601 (2010) 
${ }^{8}$ K. Saito and A. Dhar, Phys. Rev. E 83, 041121 (2011)

9 J.-S. Wang, B. K. Agarwalla, and H. Li, Phys. Rev. B 84, $153412(2011)$

10 B. K. Agarwalla, B. Li, and J.-S. Wang, Phys. Rev. E 85, 051142 (2012)

11 C. Flindt, C. Fricke, F. Hohls, T. Novotný, K. Netočný, T. Brandes, and R. J. Haug, Proc. Natl. Acad. Sci. 106, 10116 (2009)

12 Y. Utsumi, D. S. Golubev, M. Marthaler, K. Saito, T. Fujisawa, and G. Schön, Phys. Rev. B 81, 125331 (2010)

13 M. D. Ventra, Electrical Transport in Nanoscale Systems (Cambridge University Press, Cambridge, 2008)

14 H. Li, B. K. Agarwalla, and J.-S. Wang, Phys. Rev. E 86, 011141 (2012)

15 J. v. Neumann, Mathematical Fundations of Quantum Mechanics (Princeton University Press, Princeton, NJ, 1955)
16 R. B. Griffiths, Consistent Quantum Theory (Cambridge University Press, Cambridge, 2002)

17 J.-S. Wang, J. Wang, and J. T. Lü, Eur. Phys. J. B 62, 381 (2008)

${ }^{18}$ H. Haug and A.-P. Jauho, Quantum Kinetics in Transport and Optics of Semiconductors, 2nd ed. (Springer, New York, 2008)

19 G. Gallavotti and E. G. D. Cohen, Phys. Rev. Lett. 74, 2694 (1995)

20 C. Caroli, R. Combesco, P. Nozieres, and D. Saint-james, J. Phys. C: Solid St. Phys. 4, 916 (1971)

21 H. D. Cornean, P. Duclos, and R. Purice, Annales Henri Poincaré 13, 827 (2012) 